\title{
Vulnerable Plaques Producing an Acute Coronary Syndrome Exhibit a Different CT Phenotype than Those That Remain Silent
}

\author{
Răzvan-Andrei Licu ${ }^{1}$, Emanuel Blîndu ${ }^{1}$, Diana Opincariu ${ }^{1,2}$, Theodora Benedek ${ }^{1,2,3}$ \\ ${ }^{1}$ Emergency Clinical County Hospital, Târgu Mureș, Romania \\ 2 "George Emil Palade" University of Medicine, Pharmacy, Science and Technology, Târgu Mureș, Romania \\ ${ }^{3}$ Center of Advanced Research in Multimodality Cardiac Imaging, CardioMed Medical Center, Târgu Mureș, Romania
}

\section{ABSTRACT}

Background: All plaques that trigger acute coronary syndromes (ACS) present various characteristics of vulnerability. However, not all vulnerable plaques (VP) lead to an ACS. This raises the question as to which of the established CT vulnerability features hold the highest probability of developing ACS. Aim: To identify the distinct phenotype of VP that exposes the unstable atheromatous plaque to a higher risk of rupture. Material and Methods: In total, 20 patients in whom cardiac computed tomographic angiography (CCTA) identified the presence of a vulnerable plaque and who developed an ACS within 6 months after CCTA examination were enrolled in the study, and compared to 20 age- and gender-matched subjects with VPs who did not develop an ACS. All included patients presented VPs at baseline, defined as the presence of minimum 50\% degree of stenosis and at least one CT marker of vulnerability (low attenuation plaques [LAP], napkin-ring sign [NRS], positive remodeling [PR], spotty calcifications [SCs]). Results: The two groups were not different in regards to age, gender, cardiovascular risk factors, and comorbidities. Patients who developed an ACS at six months presented higher volumes of lipid-rich $(p=0.01)$ and calcified plaques $(p=0.01)$, while subjects in the control group presented plaques with a larger fibrotic content $(\mathrm{p}=0.0005)$. The most frequent vulnerability markers within VPs that had triggered ACS were LAPs ( $\mathrm{p}<0.0001)$ and PR $(\mathrm{p}<0.0001)$. Multivariate analysis identified LAP as the strongest independent predictor of ACS at 6 months in our study population (OR 8.18 [1.23-95.08], $\mathrm{p}=0.04$ ). Conclusions: VPs producing an ACS exhibit a different phenotype compared to VPs that remain silent. The CCTA profile of VPs producing an ACS includes the presence of low attenuation, positive remodeling, and lipid-rich atheroma. The presence of these features in VPs identifies very high-risk patients, who can benefit from adapted therapeutic strategies in order to prevent an ACS.

Keywords: acute coronary syndrome, risk prediction, vulnerable plaques, culprit lesions

\section{ARTICLE HISTORY}

Received: April 22, 2020

Accepted: May 30, 2020

\section{CORRESPONDENCE}

Diana Opincariu

Str. Gheorghe Marinescu nr. 38 540139 Târgu Mureș, Romania

Tel: +40 265215551

E-mail: diana.opincariu@yahoo.ro 


\section{BACKGROUND}

Acute coronary syndromes (ACS) are still associated with high morbidity and mortality despite significant advances in treatment modalities. ${ }^{1}$ According to the American Heart Association, the United States (US) had 1,413,000 hospital discharges in 2005, most of the patients suffering from non-ST-segment elevation myocardial infarction (NSTEMI) or unstable angina (UA) and just over $20 \%$ being hospitalized with ST-segment elevation myocardial infarction (STEMI). ${ }^{2}$ In addition, ACS has a high impact over the US economy, costing Americans over 150 billion dollars annually, half of which is being used on pharmacological therapies. ${ }^{3}$ Also, in the United Kingdom, more than 250,000 people develop one form of ACS each year, imposing significant costs on the National Health Service (NHS). ${ }^{4}$

ACSs are the most severe consequence of coronary atherosclerosis, being most frequently triggered by the rupture of a plaque that becomes unstable under various conditions. Coronary plaque rupture has been identified as the pathophysiological mechanism of more than $75 \%$ of fatal myocardial infarctions (MI). ${ }^{5}$ Plaques that rupture are typically characterized by a large necrotic core, with high lipid content and a thin fibrotic cap - defined as thin-cap fibroatheroma. ${ }^{6}$ However, approximately $25-40 \%$ of patients with STEMI present plaques with an intact fibrous cap. These lesions generally contain a smaller necrotic lipid core, with an intact, thick fibrotic cap. ${ }^{7}$ Three distinct pathophysiological mechanisms have been described in the process of plaque destabilization: plaque rupture (65\%), plaque erosion (30\%), and nodular calcifications $(5 \%) .{ }^{8}$ A study based on intracoronary imaging has shown that plaque rupture had triggered STEMI in $72 \%$ and NSTEMI in $32 \%$ of cases, while plaque erosion had caused STEMI in $28 \%$ versus NSTEMI in $48 \%$ of patients. In the remainder $20 \%$ of NSTEMI cases, the culprit lesion was characterized by nodular calcifications, with no signs of rupture or erosions. ${ }^{9}$ Therefore, an important question still remains regarding the reason why a coronary plaque suddenly becomes unstable and ruptures, thus causing an acute event. The identification of atherosclerotic lesions that are at high risk for rupture is of utmost importance as they can guide timely therapeutic interventions and the prevention of acute coronary events that can be possibly fatal.

The concept of vulnerable plaque (VP) has been introduced almost 30 years ago, referring to plaques that are hemodynamically insignificant, but are prone to rupture. ${ }^{10}$ Today, after the identification of other etiologies for in- travascular thrombosis, the definition of VPs has been upgraded to characterize lesions that are not as much prone to rupture, but more prone to causing an acute event. A VP is characterized by having a large lipid core, with an inflamed thin fibrous cap, positive remodeling, neoangiogenesis with intralesional hemorrhage, and also heterogeneous calcium depots. ${ }^{11}$

The identification of VPs has been the foundation for multiple cardiovascular imaging studies. Most of the vulnerability characteristics of coronary plaques can be assessed using intracoronary imaging modalities including intravascular ultrasound and optical coherence tomography. ${ }^{12,13}$ However, due to their invasive nature and inability to provide information of the overall coronary vasculature, they have limited use as a first evaluation method of patients presenting with chest pain. Coronary computed tomography angiography (CСТA) has emerged in the last years as the preferred imaging modality for investigating patients with chest pain. ${ }^{14,15}$ CCTA can noninvasively provide information regarding the coronary tree, coronary calcifications (coronary calcium score), as well as plaque morphology, composition, and vulnerability degree. CCTA markers indicative for VPs include the napkin-ring sign (NRS), low attenuation plaque (LAP), spotty calcifications (SCs), and positive remodeling (PR). ${ }^{16-20}$ The presence of these CT vulnerability markers (VM) has been associated with the risk for future acute coronary events, and various recent meta-analyses have proven their predictive capacity for ACS..$^{21,22}$

All plaques that trigger an acute coronary event present various degrees and characteristics of vulnerability. However, not all vulnerable plaques lead to an ACS. This has been suggested, on the one hand, by a systematic review of several clinical and autopsy studies, which have shown that $11.5 \%$ of patients with stable coronary artery disease present subclinical plaque rupture. ${ }^{23}$ On the other hand, Motoyama et al. (2009) have nicely shown the impact of VMs on the occurrence of ACS during follow-up. In their study cohort, from the 1,059 subjects, 15 patients with ACS had presented two $(n=45)$ or one $(n=27)$ vulnerability feature in the future culprit lesions. Despite this, the majority of patients who presented these vulnerability markers did not present an acute event (77.8\% with 2 VM and $96.3 \%$ with 1 VM, respectively). ${ }^{24}$ This raises the question as to which of the established characteristics of plaque vulnerability hold the highest probability of developing acute coronary events, thus preventing possibly catastrophic outcomes.

Therefore, we sought to evaluate VPs that had triggered an ACS in the following several months after diagnosis, 


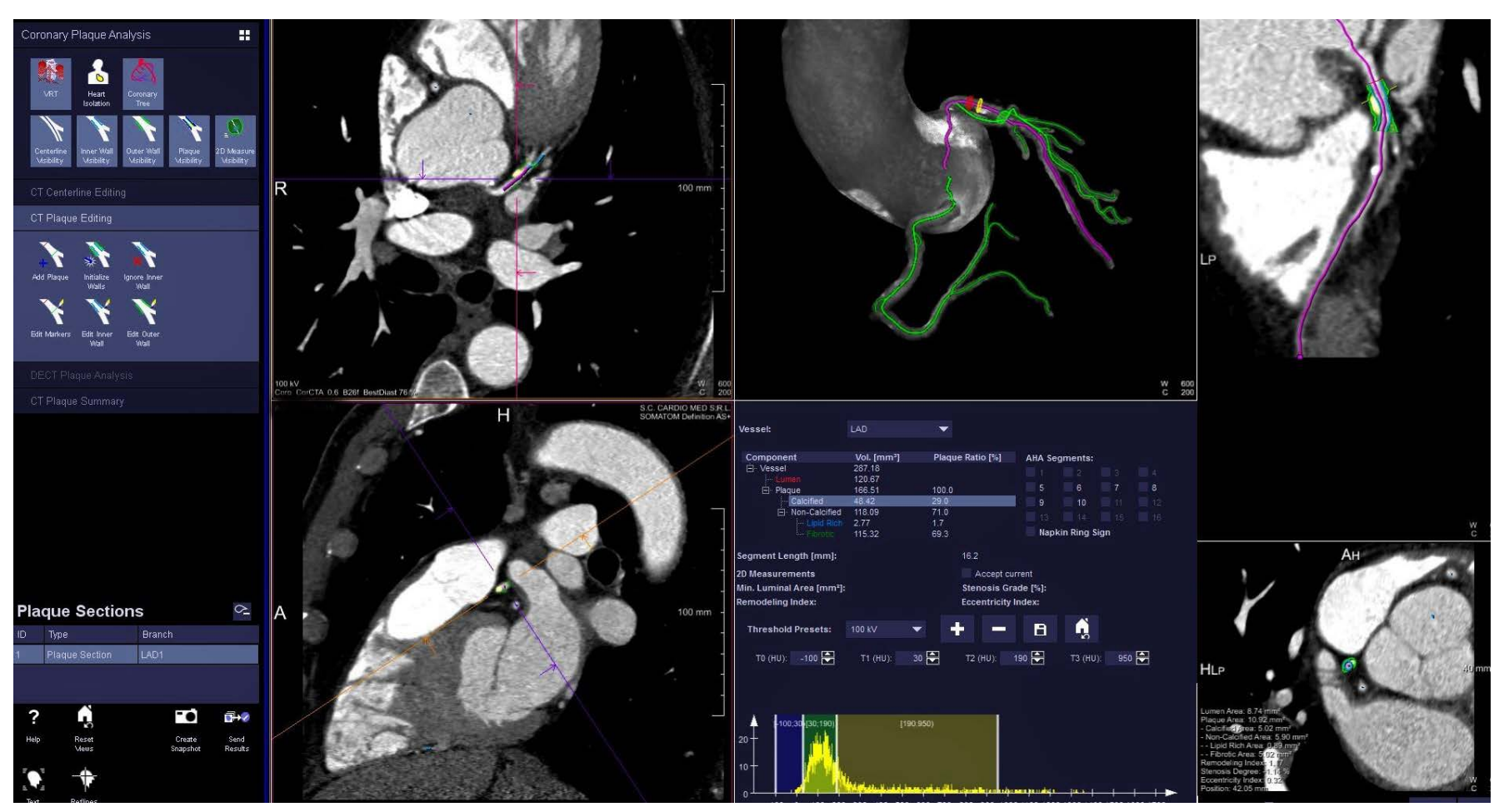

FIGURE 1. Example of a coronary plaque analysis using Syngo.via frontier software. The components of the atheromatous plaques are automatically quantified, and their volumes are displayed on the screen.

in comparison to VPs that did not cause an acute event, in patients who had undergone CCTA for chest pain. The aim of the study was to identify the distinct phenotype of VP which exposes the unstable atheromatous plaque to a higher risk of rupture.

\section{MATERIAL AND METHODS}

The study included 20 patients in whom 128 -slice CCTA performed for chest pain revealed the existence of a VP and who developed an ACS in the following 6 months after the examination, as well as 20 age- and gendermatched controls. The exclusion criteria included ACS at baseline (requiring urgent hospitalization), contraindication of administration of iodine contrast agents (acute thyroiditis, allergies), acute renal failure, or severe renal failure. Follow-up was performed by telephone, using the national healthcare database for emergency admissions. All the study procedures were performed according to good clinical practice guidelines, and the Declaration of Helsinki. Ethical approval was acquired from the institution where the study was conducted and from Ethics Committee of the "George Emil Palade" University of Medicine, Pharmacy, Science and Technology of Târgu Mureș, Romania. Written informed consent was obtained from all study subjects, prior to enrollment.
CCTA was performed using a 128-slice dual source CT (Somatom Definition, Siemens Healthcare, Germany), described in a previous study published by the research group.25 The following scan parameters were used: 120 $\mathrm{kV}$ tube voltage, gantry rotation time of $0.33 \mathrm{~s}, 128 \times 0.6$ collimation, with patients in inspiratory breath-hold position, following the same protocol. All examinations were performed at a stable heart rate below 60 beats/minute

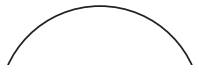

Patients with chest pain

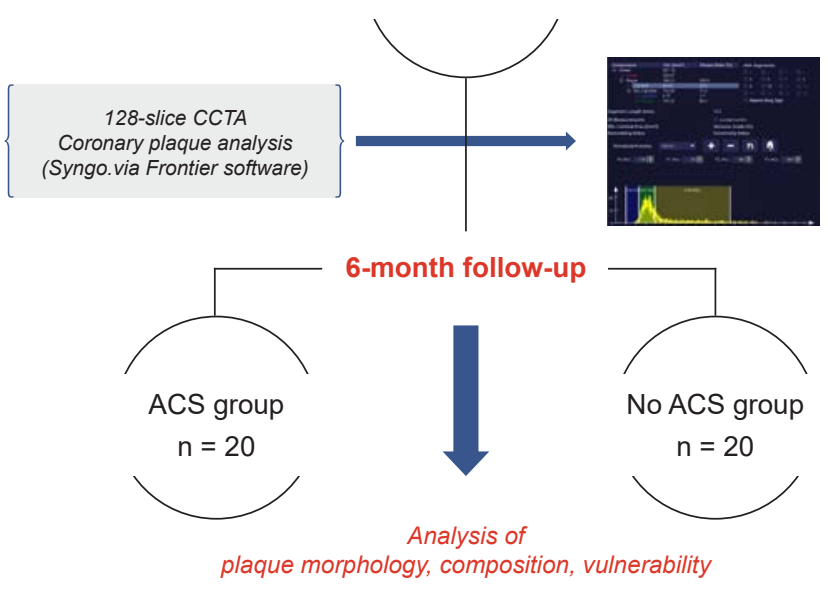

FIGURE 2. Diagram of the study protocol 
TABLE 1. Baseline characteristics of the study groups

\begin{tabular}{lccc}
\hline & $\begin{array}{c}\text { ACS group } \\
\mathbf{n = 2 0}\end{array}$ & $\begin{array}{c}\text { No ACS group } \\
\mathbf{n = 2 0}\end{array}$ & p value \\
\hline Age, yrs (median) & 65.9 & 62.35 & \\
Males, n (\%) & $13(65 \%)$ & $15(75 \%)$ & 0.16 \\
Hypertension, n (\%) & $18(90 \%)$ & $18(90 \%)$ & 1 \\
Diabetes, n (\%) & $11(55 \%)$ & $9(45 \%)$ & 0.2 \\
Dyslipidemia, n (\%) & $18(90 \%)$ & $17(85 \%)$ & 0.39 \\
Smoking, n (\%) & $4(20 \%)$ & $5(25 \%)$ & 0.49 \\
\hline
\end{tabular}

after the administration of an oral beta-blocker. Agatston coronary artery calcium score (CAC) was assessed during the pre-contrast scan, and a CAC >2,000 was considered exclusion criteria, as intense calcifications alter CCTA acquisitions. During an inspiratory breath-hold, 80-100 $\mathrm{mL}$ of iodinated contrast agent (Ultravist $370 \mathrm{mgI} / \mathrm{mL}$, Bayer Healthcare, Germany) was administered according to the patient's body weight, with a flow rate of $5.5 \mathrm{~mL} / \mathrm{s}$, followed by $50 \mathrm{~mL}$ of $0.9 \%$ saline solution at the same flow rate.

The CCTA images were post-processed for plaque analysis of morphology, composition, and detection of four CT vulnerability markers (LAP, NRS, PR, SCs). The following parameters characterizing plaque phenotypes were analyzed: stenosis (\%), plaque length ( $\mathrm{mm})$, volume (mm3), remodeling and eccentricity indexes, as well as plaque components (calcified, non-calcified, fibrotic, lipid-rich volumes and \%). Image post-processing was performed on an offline workstation with the use of Syngo.via Frontier software (Siemens Healthineers, Erlangen Germany) (Figure 1).
All included patients had presented vulnerable plaques as assessed at the baseline CCTA examination, defined as the presence of minimum $50 \%$ degree of stenosis and at least one CT vulnerability marker (LAP, NRS, PR, SCs).

A diagram of the study protocol is shown in Figure 2.

\section{RESULTS}

From the total number of patients included in the study, 20 patients presented an ACS during the 6-month followup, which were matched with 20 subjects that did not present ACS $(n=20)$. The baseline characteristics of the study population are listed in Table 1.

There were no significant differences between groups in regards to the cardiovascular risk factors and comorbidities.

The CCTA analysis of plaque morphology and composition revealed that lesions that had triggered an acute event at 6 months presented a significantly higher remodeling index $(1.13 \pm 0.35$ vs. $0.98 \pm 0.19, p=0.04)$, and higher calcified $\left(59.99 \pm 77.2 \mathrm{~mm}^{3}\right.$ vs. $35.45 \pm 77.1 \mathrm{~mm}^{3}$, $\mathrm{p}=0.01)$ and lipid-rich $\left(9.86 \pm 10.8 \mathrm{~mm}^{3}\right.$ vs. $2.68 \pm 1.0$ $\mathrm{mm}^{3}, \mathrm{p}=0.01$ ) volumes. Conversely, patients who did not exhibit an ACS had significantly larger fibrotic content in the analyzed lesions $(64.34 \pm 20.43 \%$ vs. $83.29 \pm 21.31 \%$, $\mathrm{p}=0.0005$ ) (Table 2).

When analyzing the vulnerability degree of coronary lesions, we found that patients presenting acute coronary events had a significantly higher rate of LAP ( $45 \%$ vs. $10 \%$, p <0.0001) and PR ( $50 \%$ vs. $15 \%, p<0.0001)$. However, the incidence of SCs was significantly lower in patients with ACS ( $50 \%$ vs. $65 \%, p=0.04)$. The frequency of NRS $(25 \%$

TABLE 2. CCTA evaluation of plaque morphology and composition

\begin{tabular}{lccc}
\hline & $\begin{array}{c}\text { ACS group } \\
\mathbf{n}=\mathbf{2 0}\end{array}$ & $\begin{array}{c}\text { No ACS group } \\
\mathbf{n}=\mathbf{2 0}\end{array}$ & p value \\
\hline Degree of stenosis (\%) & $53.11 \pm 10.32$ & $55.55 \pm 8.31$ & 0.41 \\
Plaque length (mm) & $17.19 \pm 5.94$ & $16.95 \pm 3.44$ & 0.95 \\
Plaque volume (mm) & $188.1 \pm 104.7$ & $186.4 \pm 90.74$ & 0.95 \\
Remodeling index & $1.13 \pm 0.35$ & $0.98 \pm 0.19$ & 0.04 \\
Eccentricity index & $0.29 \pm 0.19$ & $0.3 \pm 0.12$ & 0.87 \\
Calcified volume $\left(\mathrm{mm}^{3}\right)$ & $59.99 \pm 77.2$ & $35.45 \pm 77.1$ & 0.01 \\
Calcified \% & $27.24 \pm 22.9$ & $15.03 \pm 21.4$ & 0.02 \\
Non-calcified volume $\left(\mathrm{mm}^{3}\right)$ & $145.5 \pm 104.4$ & $151.0 \pm 73.59$ & 0.84 \\
Non-calcified \% & $72.77 \pm 22.98$ & $84.99 \pm 21.45$ & 0.02 \\
Lipid-rich volume $\left(\mathrm{mm}^{3}\right)$ & $9.86 \pm 10.8$ & $2.68 \pm 1.0$ & 0.01 \\
Lipid-rich \% & $8.42 \pm 11.8$ & $1.69 \pm 0.9$ & 0.02 \\
Fibrotic volume $\left(\mathrm{mm}^{3}\right)$ & $2.92 \pm 0.23$ & $2 \pm 0.36$ & 0.32 \\
Fibrotic \% & $64.34 \pm 20.43$ & $83.29 \pm 21.31$ & 0.0005 \\
\hline
\end{tabular}


TABLE 3. Multivariate analysis of CT vulnerability markers as predictors of ACS at 6 months

\begin{tabular}{lcc}
\hline Variable & OR $(\mathbf{9 5} \% \mathbf{C I})$ & p value \\
\hline PR & $6.44(1.07-1.97)$ & 0.0591 \\
NRS & $1.74(0.25-15.33)$ & 0.8242 \\
LAP & $8.18(1.23-95.08)$ & 0.0434 \\
SCs & $0.95(0.17-5.08)$ & 0.7793 \\
\hline
\end{tabular}

vs. $30 \%, p=0.52$ ) was not different among the two study groups (Figure 3 ).

Multivariate analysis for CT vulnerability markers identified the presence of LAP as the only independent predictor for ACS (OR 8.18 [1.23-95.08], p = 0.04).

\section{DISCUSSIONS}

The present study aimed to evaluate which vulnerability feature is most likely to cause an ACS in patients with chest pain who present VPs at the baseline CCTA evaluation. The premise of the study was not to validate the established influence of plaque vulnerability on the risk for future acute coronary events, but rather to investigate which vulnerable plaque features are more prone to trigger an ACS. This hypothesis has been suggested by previous studies that have shown a clear connection between
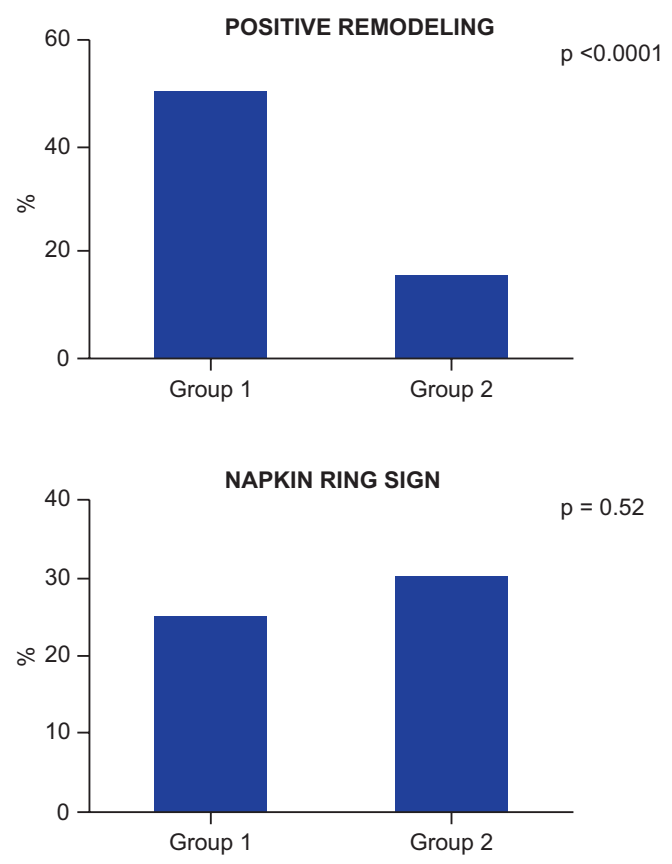

VPs and ACS, but a large number of unstable plaques, as depicted by CCTA, did not lead to an acute event. ${ }^{23,24}$ For this, we performed an analysis of patients with chest pain and VPs (defined as the presence of at least one established CCTA VM) who presented ACS, and who were matched with subjects presenting VPs at baseline, but without acute events during a 6-month follow-up period.

VPs that had triggered an acute event presented a significantly higher remodeling index. The vascular remodeling pattern at the site of an atherosclerotic lesion has shown a pivotal influence on the natural course of atherosclerosis. Outward PR is responsible for acute coronary events, while negative inward remodeling has been associated with progression of stenosis in patients with stable coronary artery disease. ${ }^{26,27}$ An increased remodeling index (>1.1) indicates the presence of PR, which is a feature of vulnerability that has been associated with other markers responsible for plaque destabilization (e.g., thin-cap fibroatheroma), and also with the risk for future cardiac events. In addition, coronary plaques that exhibit PR are more likely to have larger necrotic cores, inflamed thin fibrous caps, heterogeneous calcifications, and intralesional hemorrhages. ${ }^{19,28,29}$

Future culprit plaques exhibited higher calcified and lipid-rich content compared to plaques that remained clinically silent. On the other hand, subjects in the control
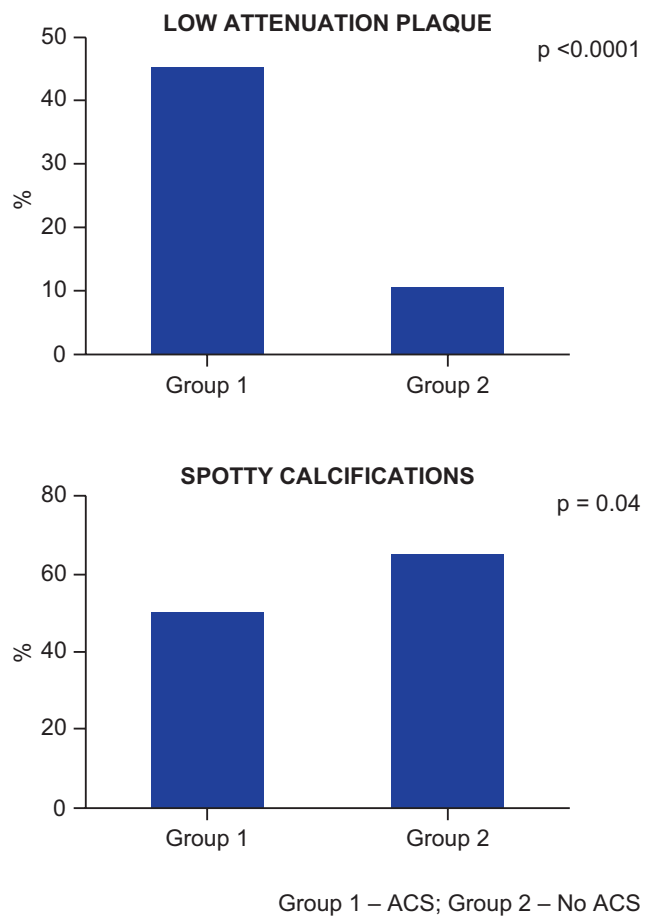

FIGURE 3. Vulnerability features present in the study groups, indicating that once identified in a coronary plaque, positive remodeling, low attenuation, and spotty calcium are associated with a higher risk for early development of an acute coronary syndrome 
group exhibited a higher fibrotic content within the analyzed plaques, which has been proven to predict plaque stability by previous studies. The study of plaque composition has led to a CCTA classification of coronary lesions into calcified, partially calcified, and non-calcified lesions. ${ }^{15,30}$ Typically, a VP has been described as a noncalcified lesion, with a large necrotic core and high lipidic content. The lipidic content of VPs is inversely correlated with the thickness of the fibrous cap, thus predicting the presence of a thin-cap fibroatheroma as evaluated via intracoronary imaging modalities. The large necrotic core $(>10 \%)$ is depicted as having a low attenuation in the CCTA examination, less than 30 Hounsfield units. ${ }^{16,20,31}$

Results in the present study indicating that future culprit plaques exhibit a higher calcified volume compare to lesions that remained clinically silent, could be considered controversial. The process of plaque mineralization is one of the final acts in the evolution of atherosclerosis, leading to plaque stabilization. However, coronary calcifications have been associated with an increased rate of adverse events by earlier CCTA studies that had focused on evaluation of coronary calcium scoring. A high calcium content may be indicative for an increased plaque burden and longer evolution of the atherosclerotic process. ${ }^{32,33}$ Despite this, a 13-month follow-up study showed that partially $(\mathrm{HR}=86.96, \mathrm{p}=0.002)$ or non-calcified $(\mathrm{HR}=58.06, \mathrm{p}=$ 0.005) plaques were associated with higher rates of major adverse cardiovascular events (MACE) compared to calcified lesions ( $\mathrm{HR}=32.94, \mathrm{p}=0.02) \cdot{ }^{34}$ Conversely, another study found that mixed and calcified lesions were more likely to predict the two-year all-cause mortality $(\mathrm{p}<0.0001) .{ }^{35}$ The divergent role of calcium may stem from the architectural deposition of calcifications within the lesion. Microcalcifications ( $<3 \mathrm{~mm}$ ) are considered as the initial marker indicating the mineralization process. Typically, these speckle-like calcium depots are actually the spotty calcifications that have been established as a CCTA feature for VP. Macrocalcifications result in sheetlike deposits ( $>3 \mathrm{~mm}$ ), which are usually characteristic for progression and stabilization of coronary lesions. Many imaging modalities have shown that spotty calcifications predict unstable plaques, while extensive, sheet-like calcium depots predict plaque stabilization. ${ }^{36}$

Coronary plaques that exhibit a higher fibrotic content and a thicker fibrous cap, are considered stable. A fibroatheroma is defined as having a lipidic pool of at least one quadrant, and if the necrotic lipidic core surpasses two quadrants, it is typically referred to as a thin-cap fibroatheroma. On the other hand, the cut-off value of $65 \mu \mathrm{m}$ is accepted for the definition of thick and thin-cap fibro- atheromas, respectively. ${ }^{6}$ Vergallo et al. found that nonculprit lesions present smaller lipidic pools with thicker fibrous caps compared to culprit lesions that had ruptured. ${ }^{37}$ The results of our study are concurrent with the observations according to which the fibrotic content is directly proportional with the stability of the plaque.

The increasing use of CCTA in cardiovascular research and also in the management of patients with chest pain and suspected coronary artery disease has led to the identification of specific high-risk criteria for VPs. The predefined CCTA features for plaque destabilization include the presence of low-attenuation plaque, spotty calcification, positive remodeling, and napkin-ring sign. ${ }^{16-20}$ Their predictive capacity for coronary risk has been nicely illustrated in a meta-analysis conducted by Nereklar et al., in which the isolated presence of each marker was associated with MACE rates (LAP: HR -2.95, p <0.001; PR: HR -2.58, p <0.001; SCs: HR 2.25, p = 0.006; NRS: HR 5.06, p <0.001). Moreover, the presence of more than two high-risk features showed additional risk in several studies, thus indicating that the more VMs are present in a coronary plaque, the higher will the risk be for presenting an ACS on the short and long term. ${ }^{21,22,24}$

Our results found that VPs that had triggered an acute event exhibited a significantly higher number of low attenuation plaques and positive outward vascular remodeling. LAP maintained its predictive capacity even after adjustments for other vulnerability markers were performed.

Low attenuation plaques, indicative for a large lipidic content, overlapped by a thin fibrous cap, have been shown to predict the rate of MACE. In the SCOT-HEART trial, LAP was the best predictor for subsequent $\mathrm{MI}$ in patients with chest pain ( $\mathrm{HR}=1.6$ per doubling, 95\% CI 1.1-2.43) over a follow-up of 4.7 years. ${ }^{38} \mathrm{~A}$ study characterizing 848 coronary plaques in 311 patients with clinical indication for CCTA has found that the total LAP volume was significantly higher in patients that had presented the composite end-point of all-cause mortality, MI, and coronary revascularization during a median follow-up of 3.2 years $(315 \pm 211 \mathrm{~mL}$ vs. $182 \pm 139 \mathrm{~mL}, \mathrm{p}=0.005) .{ }^{39}$ Moreover, a per plaque analysis on patients with ACS revealed that LAPs were present in $67.4 \%$ of culprit lesions versus only $29.03 \%$ of non-culprit lesions $(\mathrm{p}=0.0001) .{ }^{20}$

The process of outward remodeling, despite having a pivotal role in maintaining the coronary lumen unobstructed, has been linked to an increased rate of adverse events. In addition, the presence of this VM is usually accompanied by other high-risk plaque features, with incremental increase of the associated coronary risk..$^{40}$ Several large clinical trials have found that a significant propor- 
tion of patients presenting with ACS did not present a hemodynamically significant stenosis at the level of the culprit lesion, which is explained by the presence of positive remodeling. ${ }^{41-43}$

Interestingly, the presence of the napkin-ring sign was not significantly different between VPs that led to an acute event compared to those who remained clinically silent. Spotty calcifications were more frequent in plaques that did not trigger an ACS. NRS has been found as the best predictor for long-term adverse events (HR 3.85, 95\% CI 1.7-8.6, p <0.0001), together with LAP <60 HU (HR 4.96, 95\% CI 2.0-12.2, p <0.0001), even after adjustments for stenosis severity or plaque composition were made. However, SCs presented a lower predictive capacity, which was not maintained after adjustments were done. ${ }^{44}$ In a very recent study, 318 patients with mild coronary stenosis who underwent CCTA were followed up for the occurrence of MACE over a 24-month period. The results showed that both NRS $(p=0.001)$ and SCs $(p=0.027)$ were predictive for the study end-point on the univariate analysis. However, the predictive capacity of these two high-risk features was not maintained in the multivariate analysis of factors influencing the adverse events (NRS: $p$ $=0.608$, SCs: $\mathrm{p}=0.374) .45$ Their results are similar to the findings of our study, but the present analysis indicated that NRS and SCs were not associated with the occurrence of ACS at six months, both in the univariate and multivariate analysis.

The most predictive CT vulnerability feature for future ACS was the presence of LAP, indicative for a large necrotic core, which typically signals the presence of a thincap fibroatheroma. In addition to intrinsic characteristics of the vulnerable plaques, there are other factors that influence the transformation of a VP into a culprit plaque. Several other extrinsic factors of vulnerability, including inflammation and hemodynamics, have been shown to influence plaque evolution. ${ }^{11,46-49}$ Therefore, the detection of other extrinsic markers for plaque culpability (coronary shear stress, markers of local coronary inflammation) in patients presenting VPs could improve risk prediction.

\section{STUDY LIMITATIONS AND FUTURE PERSPECTIVES}

The main limits of the present study derive from the relatively low number of patients. Future patient enrollment and extension of the follow-up period could offer a better characterization of vulnerable plaques that actually lead to an acute event. This could impact the clinical approach of patients presenting with chest pain who undergo CCTA, as it can influence the therapeutic management, and thus improve outcomes, in terms of both individual patient care and quality of life, and also in terms of healthcare costs.

\section{CONCLUSIONS}

Vulnerable plaques producing an ACS exhibit a different phenotype compared to unstable plaques that remain silent. The CCTA profile of atheromatous plaques producing an ACS includes the presence of low attenuation, positive remodeling, and lipid-rich atheroma. The presence of these features in high-risk coronary plaques identifies very high-risk patients, who can benefit from adapted therapeutic strategies in order to prevent the development of an ACS.

\section{CONFLICT OF INTEREST}

None declared.

\section{ACKNOWLEDGEMENT}

This research was supported via the research grant no. 103544/2016 - PLaqueIMAGE, contract number 26/01.09.2016, financed by the Romanian Ministry of European Funds, the Romanian Government and the European Union.

\section{REFERENCES}

1. Boden WE, Shah PK, Gupta V, Ohman EM. Contemporary approach to the diagnosis and management of non-STsegment elevation acute coronary syndromes. Prog Cardiovasc Dis. 2008;50:311-351. doi: 10.1016/j.pcad.2007.11.003.

2. Rosamond W, Flegal K, Furie $\mathrm{K}$, et al. Heart disease and stroke statistics - 2008 update: a report from the American Heart Association Statistics Committee and Stroke Statistics Subcommittee. Circulation. 2008;117:e25-e146. doi: 10.1161/ CIRCULATIONAHA.107.187998.

3. Menzin J, Wygant G, Hauch O, Jackel J, Friedman M. Oneyear costs of ischemic heart disease among patients with acute coronary syndromes: findings from a multi-employer claims database. Curr Med Res Opin. 2008;24:461-468. doi: 10.1185/030079908x261096.

4. Taylor MJ, Scuffham PA, McCollam PL, Newby DE. Acute coronary syndromes in Europe: 1-year costs and outcomes. Curr Med Res Opin. 2007;23:495-503. doi: 10.1185/030079906X167462.

5. Choi SY, Mintz GS. What have we learned about plaque rupture in acute coronary syndromes? Curr Cardiol Rep. 2010;12:338343. doi: 10.1007/s11886-010-0113-X.

6. Virmani R, Kolodgie FD, Burke AP, Farb A, Schwartz SM. Lessons from sudden coronary death: a comprehensive morphological classification scheme for atherosclerotic lesions. Arterioscler Thromb Vasc Biol. 2000;20:1262-1275. doi: 10.1161/01.ATV.20.5.1262. 
7. Kajander OA, Pinilla-Echeverri N, Jolly SS, et al. Culprit plaque morphology in STEMI - an optical coherence tomography study: insights from the TOTAL-OCT substudy. EuroIntervention. 2016;12:716-723. doi: 10.4244/EIJV12I6A116.

8. Yamashita A, Asada Y. Pathology of Coronary Atherosclerotic Plaques and Mechanisms of Plaque Disruption. Ann Nucl Cardiol. 2017;3:66-72. doi: 10.17996/anc.17-00011.

9. Jia $\mathrm{H}$, Abtahian $\mathrm{F}$, Aguirre $\mathrm{AD}$, et al. In vivo diagnosis of plaque erosion and calcified nodule in patients with acute coronary syndrome by intravascular optical coherence tomography. J Am Coll Cardiol. 2013;62:1748-1758. doi: 10.1016/j. jacc.2013.05.071.

10. Muller JE, Tofler GH, Stone PH. Circadian variation and triggers of onset of acute cardiovascular disease. Circulation. 1989;79:733-743. doi: 10.1161/01.cir.79.4.733.

11. Stefanadis C, Antoniou CK, Tsiachris D, Petri P. Coronary Atherosclerotic Vulnerable Plaque: Current Perspectives. J Am Heart Assoc. 2017;6:e005543. doi: 10.1161/JAHA.117.005543.

12. Cheng JM, Garcia-Garcia HM, de Boer SP, et al. In vivo detection of high-risk coronary plaques by radiofrequency intravascular ultrasound and cardiovascular outcome: results of the ATHEROREMO-IVUS study. Eur Heart J. 2014;35:639647. doi: 10.1093/eurheartj/eht484.

13. Sinclair H, Bourantas C, Bagnall A, Mintz GS, Kunadian V. OCT for the identification of vulnerable plaque in acute coronary syndrome. JACC Cardiovasc Imaging. 2015;8:198-209. doi: 10.1016/j.jcmg.2014.12.005.

14. Knuuti J, Wijns W, Saraste A, et al. 2019 ESC Guidelines for the diagnosis and management of chronic coronary syndromes. Eur Heart J. 2020;41:407-477. doi: 10.1093/eurheartj/ehz425.

15. Rodriguez-Granillo GA, Carrascosa P, Bruining N, Waksman R, Garcia-Garcia HM. Defining the non-vulnerable and vulnerable patients with computed tomography coronary angiography: evaluation of atherosclerotic plaque burden and composition. Eur Heart J Cardiovasc Imaging. 2016;17:481491. doi: 10.1093/ehjci/jew012.

16. Kolossváry M, Szilveszter B, Merkely B, Maurovich-Horvat P. Plaque imaging with CT-a comprehensive review on coronary CT angiography-based risk assessment. Cardiovasc Diagn Ther. 2017;7:489-506. doi: 10.21037/cdt.2016.11.06.

17. Conte E, Annoni A, Pontone G, et al. Evaluation of coronary plaque characteristics with coronary computed tomography angiography in patients with non-obstructive coronary artery disease: a long-term follow-up study. Eur Heart J Cardiovasc Imaging. 2017;18:1170-1178. doi: 10.1093/ehjci/jew200.

18. Otsuka K, Fukuda S, Tanaka A, et al. Napkin-ring sign on coronary CT angiography for the prediction of acute coronary syndrome. JACC Cardiovasc Imaging. 2013;6:448-457. doi: 10.1016/j.jcmg.2012.09.016.

19. Kröner ES, van Velzen JE, Boogers MJ, et al. Positive remodeling on coronary computed tomography as a marker for plaque vulnerability on virtual histology intravascular ultrasound. Am J Cardiol. 2011;107:1725-1729. doi: 10.1016/j. amjcard.2011.02.337.

20. Benedek T, Jako B, Benedek I. Plaque quantification by coronary CT and intravascular ultrasound identifies a low CT density core as a marker of plaque instability in acute coronary syndromes. Int Heart J. 2014;55:22-28. doi: 10.1536/ihj.13-213.

21. Thomsen C, Abdulla J. Characteristics of high-risk coronary plaques identified by computed tomographic angiography and associated prognosis: a systematic review and meta-analysis.
Eur Heart J Cardiovasc Imaging. 2016;17:120-129. doi: 10.1093/ ehjci/jev325.

22. Nerlekar N, Ha FJ, Cheshire C, et al. Computed Tomographic Coronary Angiography-Derived Plaque Characteristics Predict Major Adverse Cardiovascular Events: A Systematic Review and Meta-Analysis. Circ Cardiovasc Imaging. 2018;11:e006973. doi: 10.1161/CIRCIMAGING.117.006973.

23. Arbab-Zadeh A, Fuster V. The myth of the "vulnerable plaque": transitioning from a focus on individual lesions to atherosclerotic disease burden for coronary artery disease risk assessment. J Am Coll Cardiol. 2015;65:846-855. doi: 10.1016/j. jacc.2014.11.041.

24. Motoyama S, Sarai M, Harigaya H, et al. Computed tomographic angiography characteristics of atherosclerotic plaques subsequently resulting in acute coronary syndrome. J Am Coll Cardiol. 2009;54:49-57. doi: 10.1016/j.jacc.2009.02.068.

25. Mitra N, Hodas R, Szabo E, Parajko Z, Benedek T, Benedek I. Impact of Coronary Plaque Vulnerability on Acute Cardiovascular Events - Design of a CT-based 2-year Followup Study. Journal of Interdisciplinary Medicine. 2019;4:64-71. doi: 10.2478/jim-2019-0015.

26. Schoenhagen P, Ziada KM, Kapadia SR, Crowe TD, Nissen SE, Tuzcu EM. Extent and direction of arterial remodeling in stable versus unstable coronary syndromes: an intravascular ultrasound study. Circulation. 2000;101:598-603. doi: 10.1161/01.cir.101.6.598.

27. Hong YJ, Jeong $\mathrm{MH}$, Choi $\mathrm{YH}$, et al. Positive remodeling is associated with more plaque vulnerability and higher frequency of plaque prolapse accompanied with post-procedural cardiac enzyme elevation compared with intermediate/negative remodeling in patients with acute myocardial infarction. J Cardiol. 2009;53:278-287. doi: 10.1016/j.jjcc.2008.12.006.

28. Obaid DR, Calvert PA, Brown A, et al. Coronary CT angiography features of ruptured and high-risk atherosclerotic plaques: Correlation with intra-vascular ultrasound. J Cardiovasc Comput Tomogr. 2017;11:455-461. doi: 10.1016/j. jcct.2017.09.001.

29. Motoyama S, Kondo T, Sarai M, et al. Multislice computed tomographic characteristics of coronary lesions in acute coronary syndromes. J Am Coll Cardiol. 2007;50:319-326. doi: 10.1016/j.jacc.2007.03.044.

30. van der Giessen AG, Toepker MH, Donelly PM, et al. Reproducibility, accuracy, and predictors of accuracy for the detection of coronary atherosclerotic plaque composition by computed tomography: an ex vivo comparison to intravascular ultrasound. Invest Radiol. 2010;45:693-701. doi: 10.1097/ RLI.ob013e3181e0a541.

31. Choi BJ, Kang DK, Tahk SJ, et al. Comparison of 64-slice multidetector computed tomography with spectral analysis of intravascular ultrasound backscatter signals for characterizations of noncalcified coronary arterial plaques. Am J Cardiol. 2008;102:988-993. doi: 10.1016/j. amjcard.2008.05.060.

32. Wang R, Liu X, Wang C, Ye X, Xu X, Yang C. Higher coronary artery calcification score is associated with adverse prognosis in patients with stable angina pectoris. J Thorac Dis. 2017;9:582-589. doi: 10.21037/jtd.2017.02.84.

33. Qazi AH, Zallaghi F, Torres-Acosta N, Thompson RC, O'Keefe JH. Computed Tomography for Coronary Artery Calcification Scoring: Mammogram for the Heart. Prog Cardiovasc Dis. 2016;58:529-536. doi: 10.1016/j.pcad.2016.01.007. 
34. Nance JW Jr, Schlett CL, Schoepf UJ, et al. Incremental prognostic value of different components of coronary atherosclerotic plaque at cardiac CT angiography beyond coronary calcification in patients with acute chest pain. Radiology. 2012;264:679-690. doi: 10.1148/radiol.12112350.

35. Min JK, Dunning A, Lin FY, et al. Rationale and design of the CONFIRM (COronary CT Angiography EvaluatioN For Clinical Outcomes: An InteRnational Multicenter) Registry. J Cardiovasc Comput Tomogr. 2011;5:84-92. doi: 10.1016/j. jcct.2011.01.007.

36. Mori H, Torii S, Kutyna M, Sakamoto A, Finn AV, Virmani R. Coronary Artery Calcification and its Progression: What Does it Really Mean? JACC Cardiovasc Imaging. 2018;11:127-142. doi: 10.1016/j.jcmg.2017.10.012.

37. Vergallo R, Uemura S, Soeda T, et al. Prevalence and predictors of multiple coronary plaque ruptures: In vivo 3-vessel optical coherence tomography imaging study. Arterioscler Thromb Vasc Biol. 2016;36:2229-2238. doi: 10.1161/ ATVBAHA.116.307891.

38. Williams MC, Kwiecinski J, Doris M, et al. Low-Attenuation Noncalcified Plaque on Coronary Computed Tomography Angiography Predicts Myocardial Infarction: Results From the Multicenter SCOT-HEART Trial (Scottish Computed Tomography of the HEART). Circulation. 2020;141:1452-1462. doi: 10.1161/CIRCULATIONAHA.119.044720.

39. Hadamitzky M, Jähnichen $C$, Meyer $T$, et al. Has Low Attenuation Plaque Volume in Coronary Computed Tomographic Angiography an Incremental Value For Prediction of Cardiac Events? Circulation. 2010;122:A12911. doi: 10.1161/circ.122.suppl_21.A12911.

40. Achenbach S, Ropers D, Hoffmann U, et al. Assessment of coronary remodeling in stenotic and nonstenotic coronary atherosclerotic lesions by multidetector spiral computed tomography. J Am Coll Cardiol. 2004;43:842-847. doi: 10.1016/j.jacc.2003.09.053.

41. Hoffmann U, Ferencik M, Udelson JE, et al. Prognostic Value of Noninvasive Cardiovascular Testing in Patients
With Stable Chest Pain: Insights From the PROMISE Trial (Prospective Multicenter Imaging Study for Evaluation of Chest Pain). Circulation. 2017;135:2320-2332. doi: 10.1161/ CIRCULATIONAHA.116.024360.

42. Boden WE, O'Rourke RA, Teo KK, et al. Optimal medical therapy with or without PCI for stable coronary disease. N Engl J Med. 2007;356:1503-1516. doi: 10.1056/NEJMoa070829.

43. Chang HJ, Lin FY, Lee SE, et al. Coronary Atherosclerotic Precursors of Acute Coronary Syndromes. J Am Coll Cardiol. 2018;71:2511-2522. doi: 10.1016/j.jacc.2018.02.079.

44. Feuchtner G, Kerber J, Burghard P, et al. The high-risk criteria low-attenuation plaque $<60 \mathrm{HU}$ and the napkin-ring sign are the most powerful predictors of MACE: a long-term follow-up study. Eur Heart J Cardiovasc Imaging. 2017;18:772-779. doi: 10.1093/ehjci/jew167.

45. Luo W, Zhao R, Song Y, et al. Combined non-invasive scan and biomarkers to identify independent risk factors in patients with mild coronary stenosis. J Thorac Dis. 2020;12:199-208. doi: 10.21037/jtd.2020.01.71.

46. Subirana I, Fitó M, Diaz O, et al. Prediction of coronary disease incidence by biomarkers of inflammation, oxidation, and metabolism. Sci Rep. 2018;8:3191. doi: 10.1038/s41598-01821482-y.

47. Kwiecinski J, Dey D, Cadet S, et al. Predictors of 18 F-sodium fluoride uptake in patients with stable coronary artery disease and adverse plaque features on computed tomography angiography. Eur Heart J Cardiovasc Imaging. 2020;21:58-66. doi: 10.1093/ehjci/jez152.

48. Dhawan SS, Avati Nanjundappa RP, Branch JR, et al. Shear stress and plaque development. Expert Rev Cardiovasc Ther. 2010;8:545-556. doi: 10.1586/erc.10.28.

49. Parajkó Z, Mester A, Kovács I, et al. Noninvasive Functional Characterization of Coronary Plaques by Coronary Computed Tomography - Beyond the Morphology of Vulnerable Plaques. Journal of Interdisciplinary Medicine. 2019;4:132-135. doi: 10.2478/jim-2019-0022. 